\title{
The orbital distribution of trans-Neptunian objects beyond 50 au
}

\author{
David Nesvorný ${ }^{1}$, David Vokrouhlický ${ }^{2}$, Fernando Roig ${ }^{3}$ \\ (1) Department of Space Studies, Southwest Research Institute, 1050 Walnut St., \\ Suite 300, Boulder, CO 80302, USA \\ (2) Institute of Astronomy, Charles University, V Holešovičkách 2, CZ-18000 Prague 8, \\ Czech Republic \\ (3) Observatório Nacional, Rua Gal. Jose Cristino 77, Rio de Janeiro, RJ 20921-400, \\ Brazil
}

\begin{abstract}
The dynamical structure of the Kuiper belt beyond 50 au is not well understood. Here we report results of a numerical model with long-range, slow and grainy migration of Neptune. The model implies that bodies scattered outward by Neptune to semimajor axes $a>50$ au often evolve into resonances which subsequently act to raise the perihelion distances of orbits to $q>40 \mathrm{au}$. The implication of the model is that the orbits with $50<a<100$ au and $q>40$ au should cluster near (but not in) the resonances with Neptune (3:1 at $a=62.6$ au, 4:1 at $a=75.9$ au, 5:1 at $a=88.0$ au, etc.). The recent detection of several distant Kuiper Belt Objects (KBOs) near resonances is consistent with this prediction, but it is not yet clear whether the orbits are really non-resonant as our model predicts. We estimate from the model that there should presently be $\sim 1600-2400$ bodies at the 3:1 resonance and $\sim 1000-1400$ bodies at the 4:1 resonance (for $q>40$ au and diameters $D>100 \mathrm{~km}$ ). These results favorably compare with the population census of distant KBOs inferred from existing observations.
\end{abstract}

Subject headings: Kuiper belt: general

\section{Introduction}

In our previous work, we developed a numerical model of Neptune's migration into an outer planetesimal disk (Nesvorný 2015a,b; Nesvorný \& Vokrouhlický 2016; hereafter NV16). 
By comparing the model results with the observed distribution of Kuiper belt orbits with $a<50$ au (e.g., Petit et al. 2011), we inferred that Neptune's migration must have been long-range, slow and grainy. Here we use the same model to discuss the orbital structure of the Kuiper belt beyond $50 \mathrm{au}$. We find that objects scattered by Neptune to $a>50$ au are often trapped into mean motion resonances with Neptune which act to raise the perihelion distances to $q>40 \mathrm{au}$, and detach the orbits from Neptune. The objects are subsequently released from resonances as Neptune migrates toward its present orbit. The orbital structure of the detached disk with $a>50$ au and $q>40$ au is thus expected to be clustered near Neptune's resonances. Similar results were recently reported in an independent work (Kaib \& Sheppard 2016). Section 2 briefly reviews the numerical method. The results are presented and compared with observations in Section 3. Our conclusions are given in Section 4.

\section{Method}

Integration Method. Our numerical integrations consist of tracking the orbits of four giant planets (Jupiter to Neptune) and a large number of particles representing the outer planetesimal disk. To set up an integration, Jupiter and Saturn are placed on their current orbits. Uranus and Neptune are placed inside of their current orbits and are migrated outward. The initial semimajor axis $a_{\mathrm{N}, 0}$, eccentricity $e_{\mathrm{N}, 0}$, and inclination $i_{\mathrm{N}, 0}$ define Neptune's orbit before the main stage of migration/instability. The swift_rmvs4 code, part of the Swift $N$-body integration package (Levison \& Duncan 1994), is used to follow the orbital evolution of all bodies.

The code was modified to include artificial forces that mimic the radial migration and damping of planetary orbits. These forces are parametrized by the exponential e-folding timescales, $\tau_{a}, \tau_{e}$ and $\tau_{i}$, where $\tau_{a}$ controls the radial migration rate, and $\tau_{e}$ and $\tau_{i}$ control the damping rates of $e$ and $i$ (NV16). We set $\tau_{a}=\tau_{e}=\tau_{i}$ because such roughly comparable timescales were suggested by previous work. The numerical integration is divided into two stages with migration/damping timescales $\tau_{1}$ and $\tau_{2}$ (NV16). The first migration stage is stopped when Neptune reaches $a_{\mathrm{N}, 1} \simeq 27.7$ au. Then, to approximate the effect of planetary encounters during dynamical instability, we apply a discontinuous change of Neptune's semimajor axis and eccentricity, $\Delta a_{\mathrm{N}}$ and $\Delta e_{\mathrm{N}}$. Motivated by previous results (Nesvorný \& Morbidelli 2012, hereafter NM12), we set $\Delta a_{\mathrm{N}}=0.5$ au and $\Delta e_{\mathrm{N}}=0.1$.

The second migration stage starts with Neptune having the semimajor axis $a_{\mathrm{N}, 2}=$ $a_{\mathrm{N}, 1}+\Delta a_{\mathrm{N}}$. We use the swift_rmvs4 code, and migrate the semimajor axis (and damp the eccentricity) on an e-folding timescale $\tau_{2}$. The migration amplitude was adjusted such that the planetary orbits obtained at the end of the simulations were nearly identical to the real 
orbits. This guarantees that the mean motion and secular resonances reach their present positions.

We found from NM12 that the orbital behavior of Neptune during the first and second migration stages can be approximated by $\tau_{1} \simeq 10 \mathrm{Myr}$ and $\tau_{2} \simeq 30 \mathrm{Myr}$ for a disk mass $M_{\text {disk }}=20 M_{\text {Earth }}$, and $\tau_{1} \simeq 20 \mathrm{Myr}$ and $\tau_{2} \simeq 50 \mathrm{Myr}$ for $M_{\text {disk }}=15 M_{\text {Earth }}$. The real migration slows down, relative to a simple exponential, at late stages. We therefore use $\tau_{1}=$ 10-30 Myr and $\tau_{2}=30-100 \mathrm{Myr}$. All migration simulations were run to $0.5 \mathrm{Gyr}$. They were extended to 4.5 Gyr with the standard swift_rmvs4 code (i.e., without migration/damping after 0.5 Gyr).

Migration graininess. We developed an approximate method to represent the jitter that Neptune's orbit experiences due to close encounters with massive planetesimals. The method has the flexibility to use any smooth migration history of Neptune as an input, include any number of massive planetesimals in the original disk, and generate a new migration history where the random element of encounters with the massive planetesimals is included. This approach is useful, because we can easily control how grainy the migration is while preserving the global orbital evolution of planets from the smooth simulations. See NV16 for a detailed description of the method. Here we set the mass of massive planetesimals to be equal to that of Pluto. We motivate this choice by the fact that two Pluto-class objects are known in the Kuiper belt today (Pluto and Eris). See NV16 for a discussion.

Planetesimal Disk. The planetesimal disk is divided into two parts. The part from just outside Neptune's initial orbit to $r_{\text {edge }}$ is assumed to represent the massive inner part of the disk (NM12). We use $r_{\text {edge }}=28-30$ au, because our previous simulations in NM12 showed that the massive disk's edge must be at 28-30 au for Neptune to stop at $\simeq 30$ au (Gomes et al. 2004). The estimated mass of the planetesimal disk below 30 au is $M_{\text {disk }} \simeq 15-20 M_{\text {Earth }}$ (NM12). The massive disk has a crucial importance here, because it is the main source of the resonant populations, Hot Classicals and Scattered Disk Objects (SDOs) (e.g., Levison et al. 2008). The planetesimal disk had a low mass extension reaching from 30 au to at least $\simeq 45 \mathrm{au}$. The disk extension is needed to explain why the Cold Classicals have several unique physical and orbital properties, but it does not substantially contribute to the SDOs, because of the small original mass of the extension. Here we therefore ignore the outer extension of the disk.

Each of our simulations includes one million disk particles distributed from outside Neptune's initial orbit to $r_{\text {edge }}$. The radial profile is set such that the disk surface density $\Sigma \propto 1 / r$, where $r$ is the heliocentric distance. The initial eccentricities and initial inclinations of disk particles in our simulations are distributed according to the Rayleigh distribution (Nesvorný 2015a). The disk particles are assumed to be massless, such that their gravity 
does not interfere with the migration/damping routines. This means that the precession frequencies of planets are not affected by the disk in our simulations, while in reality they were (Batygin et al. 2011).

Effects of other planets. The gravitational effects of the fifth giant planet (NM12) and planet 9 (Trujillo \& Sheppard 2014, Batygin \& Brown 2016) on the disk planetesimals are ignored. The fifth giant planet is short lived and not likely to cause major perturbations of orbits in the Kuiper belt (although this may depend on how exactly planets evolve during the instability; e.g., Batygin et al. 2012). Given its presumably wide orbit, planet 9 does not affect orbits with $a<100 \mathrm{AU}$, but may have a major influence on the structure of the scattered disk above 100 AU (e.g., Lawler et al. 2016). We therefore focus on the 50-100 au region in this work.

\section{Results}

Here we report the results of two selected simulations from NV16. The first one (Case 1) corresponds to $\tau_{1}=30 \mathrm{Myr}, \tau_{2}=100 \mathrm{Myr}, \Delta a_{\mathrm{N}}=0.5 \mathrm{au}$, and 4000 Pluto-mass objects in the original planetesimal disk. The second one (Case 2) has $\tau_{1}=10 \mathrm{Myr}, \tau_{2}=30 \mathrm{Myr}$, $\Delta a_{\mathrm{N}}=0.5 \mathrm{au}$, and 1000 Pluto-mass objects. We used a larger number of Plutos in Case 1 than in Case 2, because there is some trade off between the migration graininess and speed. Both these simulations were shown to reproduce the correct architecture of the Kuiper belt below 50 au (NV16).

Figures 1 and 2 show the orbital distribution of distant KBOs obtained in the Case-1 and Case- 2 simulations. The focus is on the orbits between 50 and $100 \mathrm{au}$. The first thing to be noted in these figures is that the distribution of orbits with $q>40$ au has a very specific structure with concentrations near Neptune's mean motion resonances (MMRs), specifically the 3:1, 4:1, 5:1 and 6:1 MMRs. Additional concentrations are seen near the 7:2, 9:2, 11:2 and weaker resonances. In Case 1, the orbits fill a semimajor axis interval that starts some 2 AU on inside of the present resonant locations (except for 6:1 MMR where the orbits are more concentrated). In Case 2, the semimajor axis distributions are more tightly concentrated near resonances. These findings are consistent with the results of Kaib \& Sheppard (2016).

The near-resonant orbits with $q>40$ au are created when the disk objects are scattered outward by Neptune and interact with resonances (Figure 3). The secular dynamics inside MMRs, mainly the Kozai cycles (Gomes 2003, Brasil et al. 2014), produce large oscillations

of $e$ and $i$. When Neptune is still migrating, these resonant objects can be released from resonances with $q>40$ au and remain on stable orbits in the detached disk. The vast 
majority of these orbits are not inside the resonances today (the resonant angles do not librate) 1

The resonant fingers shown in Figures 1 and 2 are a specific prediction of a model with the slow migration of Neptune (Nesvorný 2015a). The fast migration $(\tau<10 \mathrm{Myr})$ does not produce these fingers because there is not enough time with the fast migration for the secular cycles to act to raise the perihelion distance. Then, when Neptune stops migrating, all captures in resonances become temporary and the perihelion distances do not drop below 40 AU. Furthermore, the high-eccentricity phase of Neptune, investigated by Levison et al. (2008), would produce a different structure of the detached disk with $q>40 \mathrm{au}$, where there is no strong preference for the resonant orbits.

The recent detection of several new KBOs with $50<a<100$ au and $q>40$ au (Sheppard et al. 2016) are in line with our model predictions. These objects tend to concentrate toward resonances. There is 2015 FJ345, 2013 FQ28 and 2015 KH162 at the 3:1 resonance, 2014 FZ71 and 2005 TB190 at the 4:1 resonance and 2008 ST291 at the 6:1 resonance (Sheppard et al. 2016). In addition, all these objects, except of 2012 FH84, have high orbital inclinations $\left(i>20^{\circ}\right)$, as expected if the Kozai cycles played role in their origin. We find from our simulations that the orbits with $q>40$ au indeed have large inclinations (characteristically $\simeq 25-45 \mathrm{deg}$, with a clear correlation between $q$ and $i$; Figure 4). This provides additional support for our model. The mean inclination of orbits with $q<40$ au is $25.7^{\circ}$ in Case 1 and $21.3^{\circ}$ in Case 2. The mean inclination of orbits with with $q>40$ au is similar $\left(\simeq 35^{\circ}\right)$ in both cases. The slower and grainier migration in Case 1 produced several low-inclination orbits $\left(i \lesssim 10^{\circ}\right)$ with $q>40$ au, while these orbits are almost non-existent in Case 2.

The orbits of distant KBOs with $q>40$ au are not known well enough to establish whether they are resonant (which would contradict predictions of our model) or non-resonant (which would support our model). Future observations will help to resolve this issue. In addition, the semimajor axis distributions of objects with $q>40$ au are sensitive to Neptune's migration speed with faster migrations speeds implying more concentrated populations. This can be used, when the distributions are well characterized by observations, as a diagnostic

\footnotetext{
${ }^{1}$ Here we opt for not discussing the 5:2 resonance in detail, mainly because there is still some disagreement about how large the population of objects inside the 5:2 resonance actually is (e.g., Volk et al. 2016, Sheppard et al. 2016). A large number of objects end up near the 5:2 resonance in our simulations (Figures 1 and2). A careful analysis shows that only a fraction of these objects are inside the 5:2 resonance today ( $\simeq 50$ particles in both the Case-1 and Case-2 simulations show sustained 5:2 resonant librations in an extended 10-Myr simulation). This indicates the 5:2 implantation efficiency $\simeq 5 \times 10^{-5}$, roughly $1 / 4$ of the $3: 2$ implantation efficiency in Case 1 with 4000 Plutos (NV16).
} 
of Neptune's migration speed (Kaib \& Sheppard 2016). Unlike the inclination distribution considered in Nesvorný (2015a), which can be used to mainly constrain the early stages of Neptune's migration, the semimajor axis distributions considered here should be more sensitive to the migration speed (and graininess) during the last $\sim 1$ au of Neptune's migration. If the independent arguments derived from Saturn's obliquity are valid (e.g., Vokrouhlický \& Nesvorný 2015), Neptune's migration was very slow during the late stages ( $\tau \sim 150$ Myr $)$, thus favoring Case 1 over Case 2, and the semimajor axis distributions that are more spread on the inner side of resonances.

In the nomenclature of Gladman et al. (2008), the SDOs can be divided into scattering objects (the ones that are currently scattering actively off Neptune; e.g., (15874) 1996 TL $_{66}$, Luu et al. 1997), and detached objects as being non-scattering SDOs with large eccentricity (e.g., (148209) $2000 \mathrm{CR}_{105}$, Buie et al. 2000). The scattering objects, defined as those whose semimajor axis changed more than 1.5 au in 10 Myr (Gladman et al. 2008), are denoted by red dots in Figures 1 and 2 . We find that the slow migration model $(\tau \gtrsim 10 \mathrm{Myr})$ implies that the detached population should represent the majority of SDOs. Specifically, the implantation efficiency as a detached object with $50<a<100$ au is $2.0 \times 10^{-3}$ in both Case-1 and Case-2 simulations (Table 1). The implantation efficiency as a scattering object is much smaller, $3.7 \times 10^{-4}$ in Case 1 and $4.6 \times 10^{-4}$ in Case 2. This shows that the detached population should be $\simeq 5$ times larger than the scattering population. All estimates reported here apply to the part of the scattered disk between 50 and $100 \mathrm{au}$.

Nesvorný et al. (2013) estimated, using their model of Jupiter Trojan capture and the current population of Trojans, that the original planetesimal disk should have contained $\sim 2 \times 10^{7}$ bodies with diameters $D>100 \mathrm{~km}$ (this assumes $7 \times 10^{-7}$ Trojan capture efficiency and the fact that there are 15 Jupiter Trojans with $H<8.7$, which corresponds to $D>100 \mathrm{~km}$ for a $6 \%$ albedo). If so, the detached population with $50<a<100$ au should have $\sim 40,000$ objects with $D>100 \mathrm{~km}$. The scattering population in the same semimajor axis range should be smaller $(\sim 8,000$ objects with $D>100 \mathrm{~km})$. A careful consideration of observation biases will be required to understand how well this corresponds to the reality.

Sheppard et al. (2016) estimated that there are $2400_{-1000}^{+1500}$ and $1600_{-1200}^{+2000}$ objects with $q>40$ au and $D>100 \mathrm{~km}$ at the $3: 1$ and 4:1 resonances. From our simulations, assuming $2 \times 10^{7} D>100 \mathrm{~km}$ objects in the original disk and the implantation efficiencies reported in Table 1, we compute that there should be between $\sim 1600$ (for Case 2) and $\sim 2400$ (for Case 1) objects with $D>100 \mathrm{~km}$ at the $3: 1$ resonance, and between $\sim 1000$ (for Case 2) and $\sim 1400$ (for Case 1 ) objects with $D>100 \mathrm{~km}$ at the $4: 1$ resonance. This is consistent with the findings of Sheppard et al. (2016). The populations are larger with slower migration (Case 1) because this case allows more time for the implantation of bodies into the detached disk. 
This dependence could, in principle, be used to constrain the migration speed of Neptune. For that, however, we would need to consider a larger suite of integrations and have better observational constraints. According to our model, somewhat smaller populations should exists near the 5:1 and 6:1 resonances $(\sim 500-1000$ with $D>100 \mathrm{~km}$ and $q>40$ au), and this trend should continue to weaker resonances beyond $100 \mathrm{au}$.

\section{Conclusions}

Our simulations with slow migration of Neptune (as required from the inclination constraint; Nesvorný 2015a) lead to the formation of a prominent detached disk with substantial populations of objects concentrated at various MMRs with Neptune. This is an important prediction of the model, which is testable by observations. The current surveys are only starting to have a sufficient sensitivity to probe the orbital distribution of bodies with large perihelion distances (e.g., Shankman et al. 2016).

Sheppard et al. (2016) reported several new objects in the detached disk between 50 and $100 \mathrm{au}$. They found that these objects are near Neptune's MMRs and have significant inclinations $\left(i>20^{\circ}\right)$. Interestingly, these findings are consistent with the predictions of our model with slow migration of Neptune. The population census of near-resonant SDOs inferred from observations is also consistent with the model.

Our results imply that the detached population at 50-100 au should be $\simeq 5$ times larger than the scattering population in the same semimajor axis range, which may have important implications for the origin of Jupiter-family comets. In addition, there seems to be a large population of objects with $q \simeq 35-40$ au in the 5:1 MMR (Pike et al. 2015), which cannot be easily explained by the resonant sticking of scattering objects (Yu et al. 2015). Instead, we find it possible that these objects are the low- $q$, easier-to-detect part of the resonant populations that continue to $q>40$ au.

The work of D.N. was supported by NASA's Emerging Worlds program and Brazil's Science without Borders program. D.V. was supported by the Czech Grant Agency (grant GA13-01308S). F.R. was supported by Brazil's Council of Research. All CPU-expensive simulations in this work were performed on NASA's Pleiades Supercomputer. We thank Nathan Kaib and Scott Sheppard for discussions, and for making it possible to submit our article simultaneously with theirs (Kaib \& Sheppard 2016). We also thank an anonymous reviewer for useful suggestions. 


\section{REFERENCES}

Batygin, K., Brown, M. E., \& Betts, H. 2012, ApJ, 744, L3

Batygin, K., Brown, M. E., \& Fraser, W. C. 2011, ApJ, 738, 13

Batygin, K., \& Brown, M. E. 2016, AJ, 151, 22

Brasil, P. I. O., Gomes, R. S., \& Soares, J. S. 2014, A\&A, 564, A44

Gladman, B., Marsden, B. G., \& Vanlaerhoven, C. 2008, The Solar System Beyond Neptune, 43

Gomes, R. S. 2003, Icarus, 161, 404

Gomes, R. S., Morbidelli, A., \& Levison, H. F. 2004, Icarus, 170, 492

Kaib, N. A., \& Sheppard, S. S. 2016, arXiv:1607.01777

Lawler, S. M., Shankman, C., Kaib, N., et al. 2016, arXiv:1605.06575

Levison, H. F., \& Duncan, M. J. 1994, Icarus, 108, 18

Levison, H. F., Morbidelli, A., Vanlaerhoven, C., Gomes, R., \& Tsiganis, K. 2008, Icarus, 196,258

Luu, J., Marsden, B. G., Jewitt, D., et al. 1997, Nature, 387, 573

Nesvorný, D. 2015a, AJ, 150, 73

Nesvorný, D. 2015b, AJ, 150, 68

Nesvorný, D., \& Morbidelli, A. 2012 (NM12), AJ, 144, 117

Nesvorný, D., \& Vokrouhlicky, D. 2016 (NV16), arXiv:1602.06988

Nesvorný, D., Vokrouhlický, D., \& Morbidelli, A. 2013, ApJ, 768, 45

Petit, J.-M., Kavelaars, J. J., Gladman, B. J., et al. 2011, AJ, 142, 131

Pike, R. E., Kavelaars, J. J., Petit, J. M., et al. 2015, AJ, 149, 202

Shankman, C., Kavelaars, J., Gladman, B. J., et al. 2016, AJ, 151, 31

Sheppard, S. S., Trujillo, C., \& Tholen, D. J. 2016, ApJ, 825, L13

Trujillo, C. A., \& Sheppard, S. S. 2014, Nature, 507, 471 
Vokrouhlický, D., \& Nesvorný, D. 2015, ApJ, 806, 143

Volk, K., Murray-Clay, R., Gladman, B., et al. 2016, AJ, 152, 23

Yu, T. Y. M., Murray-Clay, R., \& Volk, K. 2015, AAS/Division for Planetary Sciences Meeting Abstracts, 47, 211.08

This preprint was prepared with the AAS IATEX macros v5.2. 


\begin{tabular}{lrr}
\hline \hline & $\begin{array}{r}\text { Case 1 } \\
\left(\times 10^{-4}\right)\end{array}$ & $\begin{array}{r}\text { Case 2 } \\
\left(\times 10^{-4}\right)\end{array}$ \\
\hline Detached & 20 & 20 \\
Scattering & 3.7 & 4.6 \\
$3: 1$ & 1.1 & 0.78 \\
$4: 1$ & 0.70 & 0.47 \\
$5: 1$ & 0.48 & 0.24 \\
$6: 1$ & 0.32 & 0.26 \\
\hline \hline
\end{tabular}

Table 1: The implantation probabilities in various target regions. These estimates were obtained by determining the number of particles that ended in target regions at $4.5 \mathrm{Gyr}$ and dividing it by the number of particles in the original disk $\left(10^{6}\right)$. The scattering objects are defined as those with the semimajor axis change $\Delta a>1.5$ au in a 10 Myr integration. The detached objects have more stable orbits $(\Delta a<1.5 \mathrm{au})$. The estimates are given for the populations with $50<a<100 \mathrm{au}$. The resonant populations include objects both inside and close to the resonances with the latter ones being overhelmingly more common. The identification of these objects in the distributions shown in Figures 1 and 2 was straightforward (we used appropriate semimajor axis ranges). 


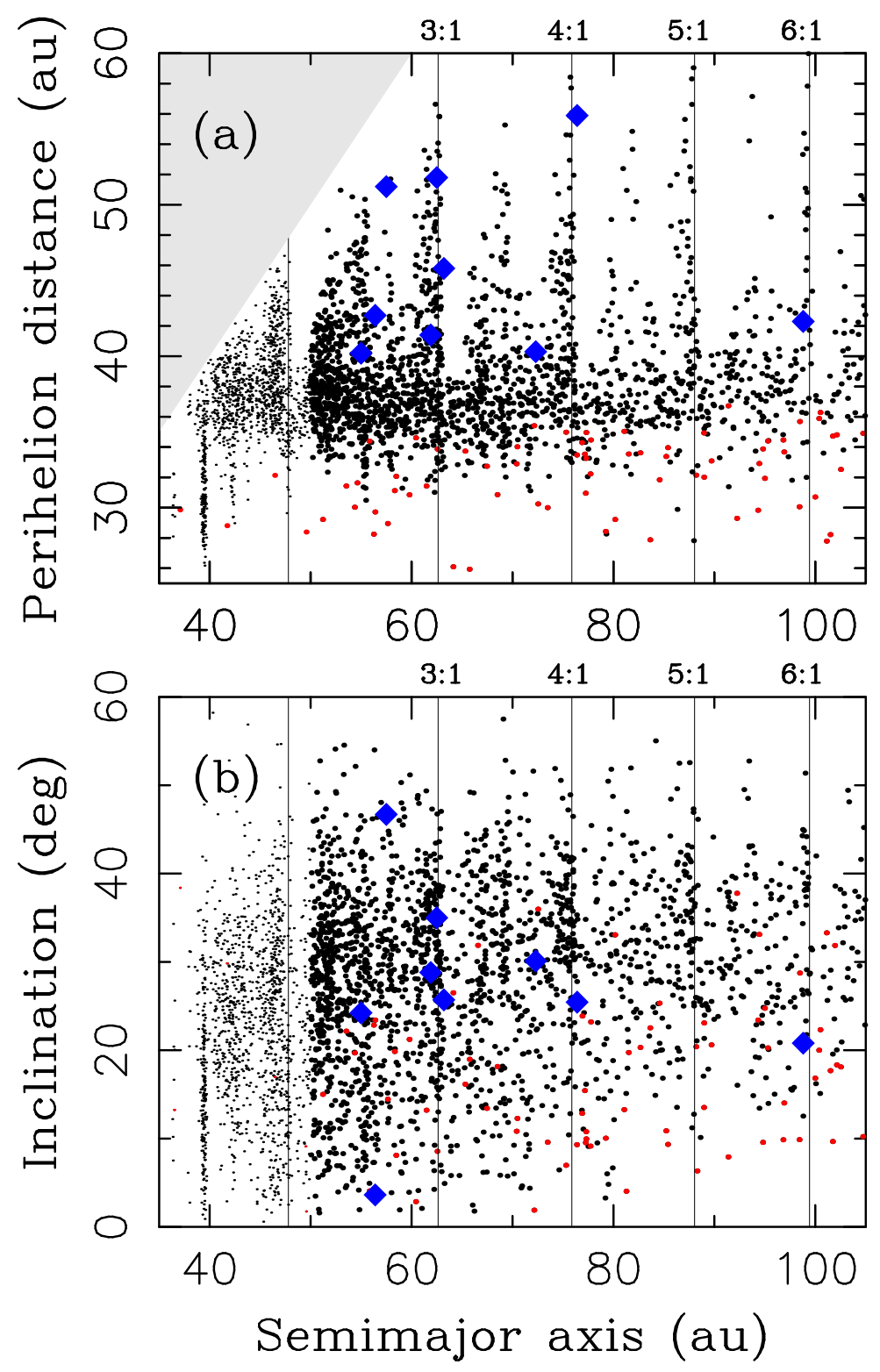

Fig. 1. - The orbital distribution of bodies produced in our Case-1 simulation $\left(\tau_{1}=30 \mathrm{Myr}\right.$, $\tau_{2}=100 \mathrm{Myr}, 4000$ Plutos). The upper (lower) panel shows the perihelion distance (inclination). The orbits with $a>50 \mathrm{au}$, which are the main focus here, are denoted by larger dots. The scattering orbits, defined as those whose semimajor axis changed more than 1.5 au in a 10-Myr integration (Gladman et al. 2008), are denoted by red dots. Note the massive detached population (black dots). The detached objects with $q>40$ au are concentrated near resonances. The known KBOs with $q>40 \mathrm{au}$, reported in Table 1 of Sheppard et al. (2016), are shown by blue diamonds. The orbital elements plotted here are barycentric. 

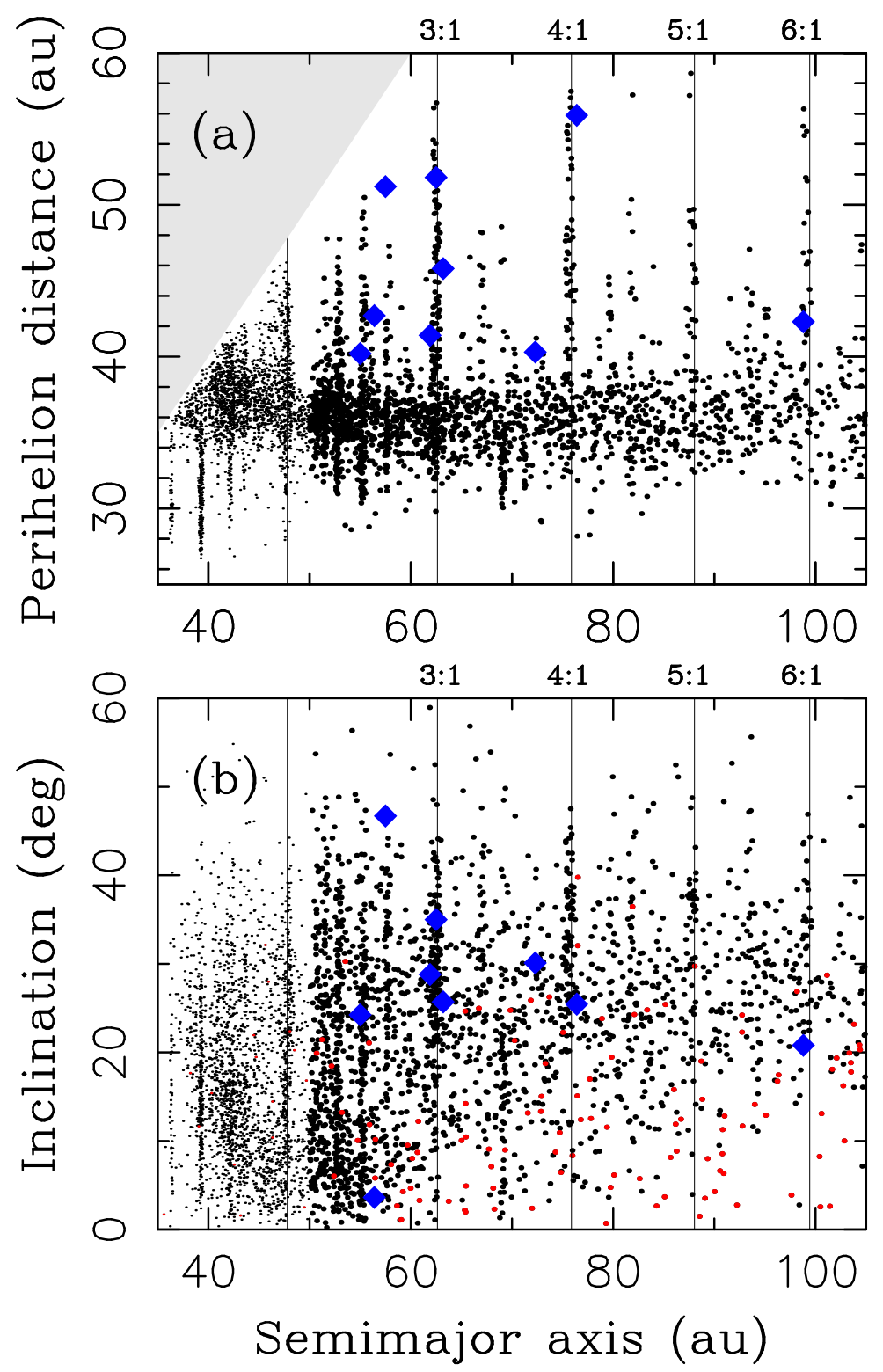

Fig. 2.- The same as Figure 1 but for the Case-2 simulation $\left(\tau_{1}=10 \mathrm{Myr}, \tau_{2}=30 \mathrm{Myr}\right.$, 1000 Plutos). 

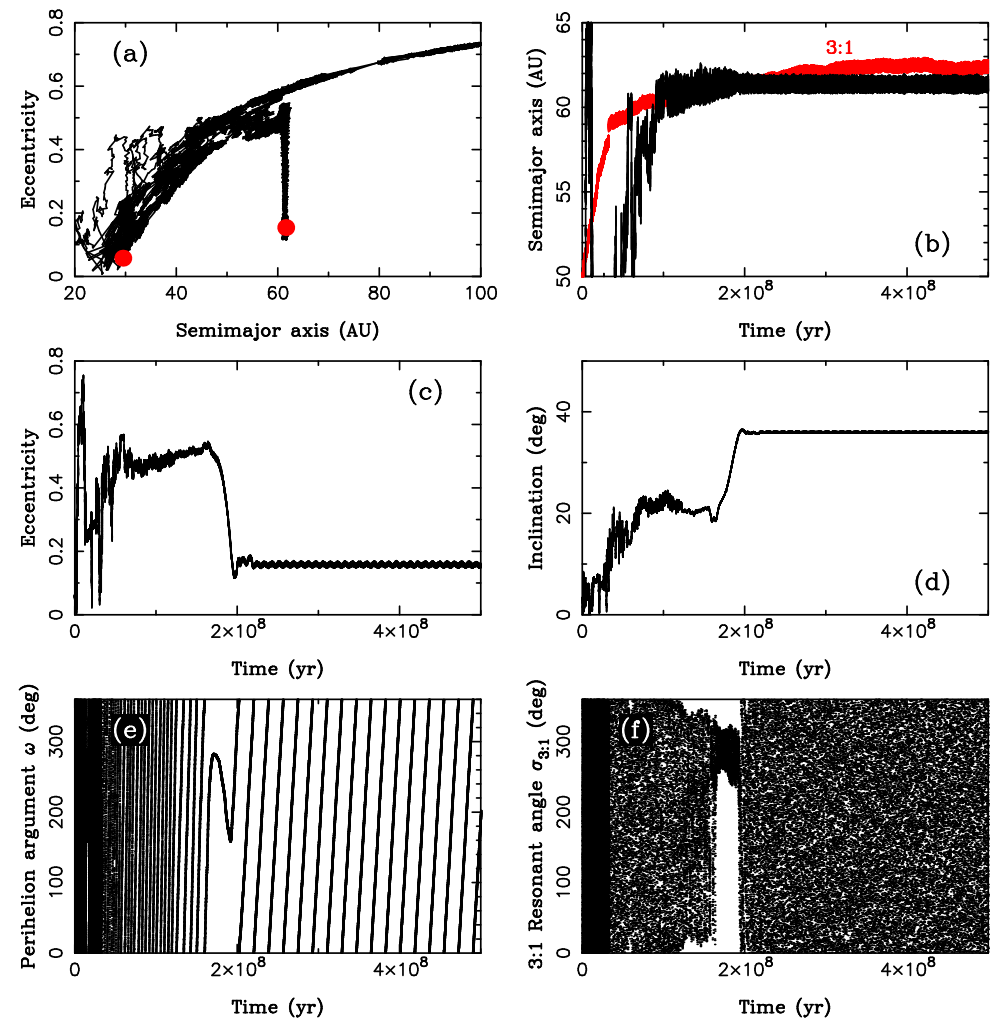

Fig. 3.- An example of orbital evolution that ended with a detached orbit near the 3:1 MMR with Neptune. The red dots in panel (a) show the initial $\left(a=29.47 \mathrm{au}, e=0.058, i=3.4^{\circ}\right)$ and final orbit $\left(a=61.24 \mathrm{au}, e=0.160, i=36.0^{\circ}\right)$. During the first stage of integration, the body is scattered by Neptune to an orbit with $a>50 \mathrm{au}, i \simeq 20^{\circ}$ (panel d) and large eccentricity (panel c). It subsequently becomes trapped in the 3:1 MMR with Neptune, shown by the red line in panel $\mathrm{b}$. The libration of the resonant angle $\sigma_{3: 1}=3 \lambda-\lambda_{N}-2 \varpi$, where $\lambda$ and $\lambda_{N}$ are body's and Neptune's mean longitudes, and $\varpi$ is the perihelion longitude, occur between $t=180$ and $200 \mathrm{Myr}$ (panel f). The resonant orbit is affected by Kozai cycles (panel e) during which the eccentricity decreases and inclination increases, and the orbit decouples from Neptune. Finally, since Neptune is migrating, the orbit drops from the 3:1 $\mathrm{MMR}$ and ends up $\simeq 1.4$ au below the present resonance $(a=62.6 \mathrm{AU})$. 

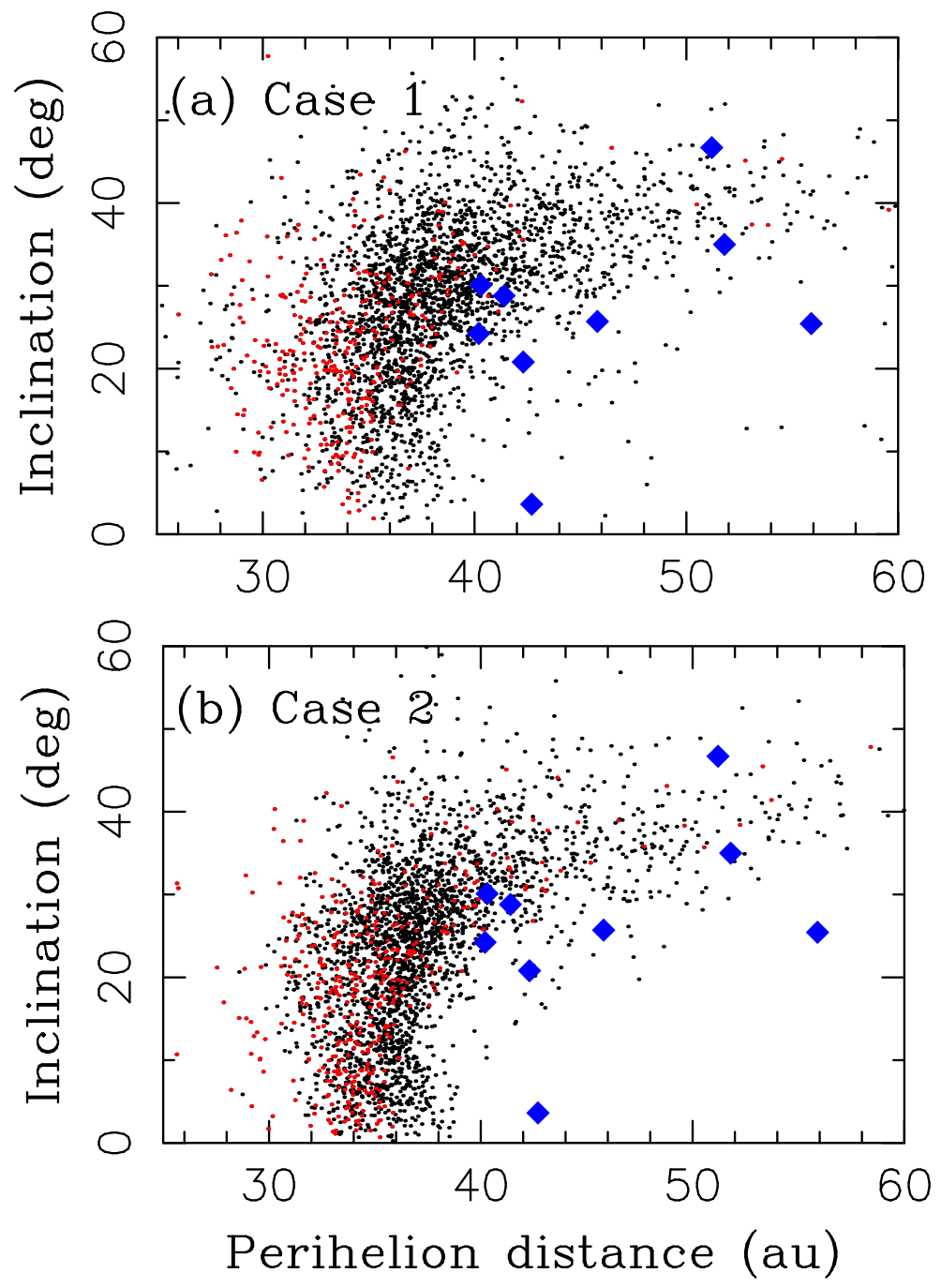

Fig. 4. - The inclination distribution of distant KBOs $(a>50 \mathrm{au})$ obtained in the Case 1 (a) and Case 2 (b) simulations. While the model objects with $q<40$ au have a wide range of inclinations, the ones with $q>40$ au generally have $i>20^{\circ}$. This corresponds pretty well to the orbital inclinations of KBOs detected by Sheppard et al. (2016) (here shown by blue diamonds). The KBO with $q=42.7$ au and $i=3.6^{\circ}, 2012 \mathrm{FH} 84$, was probably not produced by the mechanism discussed here. Instead, it may trace a continuation of Cold Classicals beyond 50 au (Sheppard et al. 2016). The scattering orbits are denoted by red dots. 